\title{
DYNAMICS, CONTROL, AND STABILIZATION OF TURNING FLIGHT IN FRUIT FLIES
}

\author{
LEIF RISTROPH $(\bowtie)^{*}$, ATTILA J. BERGOU ${ }^{\dagger}$, GORDON J. BERMAN $^{\ddagger}$, \\ JOHN GUCKENHEIMER ${ }^{\S}$, Z. JANE WANGף, AND ITAI COHEN"
}

\begin{abstract}
Complex behaviors of flying insects require interactions among sensory-neural systems, wing actuation biomechanics, and flapping-wing aerodynamics. Here, we review our recent progress in understanding these layers for maneuvering and stabilization flight of fruit flies. Our approach combines kinematic data from flying insects and aerodynamic simulations to distill reduced-order mathematical models of flight dynamics, wing actuation mechanisms, and control and stabilization strategies. Our central findings include: (1) During in-flight turns, fruit flies generate torque by subtly modulating wing angle of attack, in effect paddling to push off the air; (2) These motions are generated by biasing the orientation of a biomechanical brake that tends to resist rotation of the wing; (3) A simple and fast sensory-neural feedback scheme determines this wing actuation and thus the paddling motions needed for stabilization of flight heading against external disturbances. These studies illustrate a powerful approach for studying the integration of sensory-neural feedback, actuation, and aerodynamic strategies used by flying insects.
\end{abstract}

Key words. Insect flight, aerodynamics, flight dynamics, control, stability

AMS(MOS) subject classifications. 37N25, 76Z10, 92B05, 70E99

1. Introduction. The flight of insects is a beautiful example of an organism's complex interaction with its physical environment. Consider, for example, a fly's evasive dodge of an approaching swatter. The insect must orchestrate a cascade of events that starts with the visual system perceiving information that is then processed and transmitted through neural circuits. Next, muscle actions are triggered that induce changes to the insect's wing motions, and these motions interact with fluid flows to generate aerodynamic forces. As another example, even the simple task of flying straight requires similarly complex events in order to overcome unexpected disturbances and suppress intrinsic instabilities. Here, we review our recent progress in dissecting the many layers that comprise maneuvering and stabilization in the flight of the fruit fly, D. melanogaster [1-3]. Our emphasis is on aspects of flight at the interface of biology and physics, and we seek to understand how physical effects both constrain and simplify biological strategies.

*Department of Physics, Cornell University, Ithaca, NY 14853, USA, lgr24@cornell.edu. The authors thank the NSF for support.

${ }^{\dagger}$ Department of Engineering, Brown University, Providence, RI 55555, USA

${ }^{\ddagger}$ Lewis-Sigler Institute for Integrative Genomics, Princeton University, Princeton, NJ 08544, USA

$\S$ Department of Mathematics, Cornell University, Ithaca, NY 14853, USA

๑Departments of Mechanical and Aerospace Engineering and Physics, Cornell University, Ithaca, NY 14853, USA

" Department of Physics, Cornell University, Ithaca, NY 14853, USA

S. Childress et al. (eds.), Natural Locomotion in Fluids

and on Surfaces, IMA 155, DOI 10.1007/978-1-4614-3997-4_6,

(C) Springer Science+Business Media New York 2012 
Over the last 40 years, turning flight in insects has emerged as an archetype of complex animal behaviors [4-12]. When searching for food, flies exhibit a stereotyped exploratory behavior in which straight flight paths are separated by rapid turns called saccades. Fruit flies turn when triggered by specific visual stimuli, and a typical saccade through $90^{\circ}$ is completed in $50 \mathrm{~ms}$ or about 10 wing-beats [13]. Given that a blink of an eye is about $250 \mathrm{~ms}$, these maneuvers are quite impressive. Is it difficult for a fly to perform a saccade? Physically, one might address this question by comparing the torque needed to turn its body with the torque exerted simply to keep the body aloft. The scale of the turning torque is given by the body moment of inertia times angular acceleration: $I \alpha \approx\left(10^{-13} \mathrm{~kg} / \mathrm{m}^{2}\right)\left(90^{\circ} /(50 \mathrm{~ms})^{2}\right) \approx 10^{-10} \mathrm{Nm}$. To hold its milligram body up during hovering, the millimeter-scale wings exert torques of about $M g r \approx\left(10^{-6} \mathrm{~kg}\right)\left(10 \mathrm{~m} / \mathrm{s}^{2}\right)(1 \mathrm{~mm}) \approx 10^{-8} \mathrm{Nm}$. This simple estimate shows that the torque needed to turn is only a few percent of the torque produced during hovering. Thus, counter-intuitively, even these extreme flight maneuvers are achieved with little additional effort.

However, what appears to be effortless in terms of torque exertion is difficult in nearly all other respects. For example, the changes in wing motions needed to induce such a maneuver are also expected to be a few percent [9], which amounts to adjustments in wing orientation on the scale of a few degrees! What modulations to wing motions do insects actually make, and how small are these changes? Of course, such minuscule adjustments demand precise muscular actuation [14]. How do muscle actions lead to subtle modulations of wing motions? Further, the time-scales involved in such maneuvers are so fast that the turn is often complete within the visual system reaction time [7]. How are these wing motions orchestrated if the insect is effectively blind during the maneuver? Armed with knowledge of the force scales, one can now also appreciate the difficulty of simply maintaining straight flight. Air is a messy environment, and small external torque or noise in the flight motor will knock the insect off its intended path. How do fruit flies resist unwanted body rotations and keep on-course?

In this work, we take a tour through some of these aspects of turning behavior of fruit flies. We show that analyzing motions of flying insects reveals a remarkable amount of information about sensory, neural, muscular, and aerodynamic processes. First, we review recent developments in techniques for motion tracking of flying insects and in modeling aerodynamic forces on flapping wings. We apply these tools to reveal how subtle adjustments to wing motions drive turning maneuvers. Next, we go a level deeper to examine how the wing motions themselves emerge from the interaction of muscular actuation, aerodynamic forces, and biomechanics of the wing hinge. Finally, we examine the fruit fly's "auto-pilot", a sensory-neural scheme that uses feedback to maintain body orientation during straight flight. By combining results from each of these studies, we demonstrate 
a

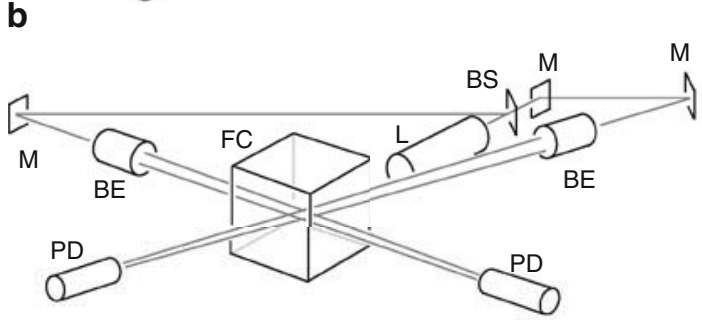

C

LA

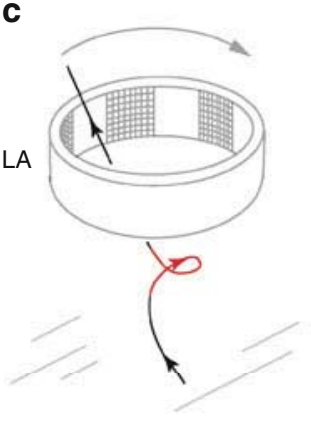

d

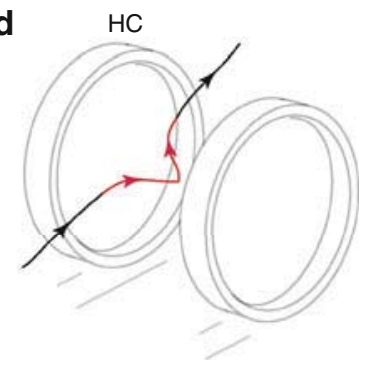

FIG. 1. Schematics of the experimental set-ups [1-3]. (a) Three orthogonal highspeed cameras capture the flight of insects within a clear flight chamber. Each camera is back-lit by a single bright light-emitting diode focused with a lens. (b) An automatic trigger consists of crossed laser beams to detect the presence of an insect in the filming volume and initiate recording. (c) A computer-controlled array of lights presents insects with rotating striped patterns and reliably generates turning maneuvers. (d) Helmholtz coils generate a field that applies a torque to a magnetic pin glued to the back of an insect, thus disrupting its flight

that complex flight behaviors can be understood in terms of the integration of reduced-order mathematical models.

\section{Experimental Methods: Videography, Behavioral} Stimulation, and Motion Tracking. Given that adjustments to wing motions are expected to be subtle, our experimental emphasis is on developing precision techniques for gathering large quantities of flight data. Here, we present three experimental advances needed to address flight maneuverability and stability. First, we show how to automate the high-speed video capture process in order to obtain many flight sequences. Second, we elicit specific behaviors by presenting insects with visual stimuli and by mechanically perturbing their flight. Third, we outline our algorithm for automatically extracting wing and body motion data from flight videos. 
We have assembled an automated, versatile system for capturing many high-speed video sequences of free-flying insects [1]. As shown in Fig. 1a, three high-speed cameras are focused on a cubical filming volume contained within a large clear flight chamber. To provide sufficient light, each camera is back-lit so that the insects appear as silhouettes in our videos. Typically, many insects are released into this chamber, and recording is automatically triggered by their presence in the filming volume. The automatic trigger consists of two laser beams that intersect in this region (Fig. 1). When the beams are simultaneously broken, this event is detected with photodiodes and an electronic circuit initiates recording of the cameras. After recording, the cameras automatically become available to film another event, allowing us to capture many sequences.

To capture many movies of rare events such as turning flight, it is necessary to stimulate the behavior within the filming volume. To initiate turning maneuvers, we take advantage of a well-known behavior of fruit flies [15]: when presented with a moving object or pattern, these insects tend to fixate the object by turning with it. We assembled an arena in which rotating light patterns can be played on a circular array of lightemitting diodes, as shown in Fig. 1c. We use the laser trigger signal to initiate rotation of a light-dark striped pattern, yielding saccadic turns [3].

To study flight stability, we devised a complementary system that imposes mechanical perturbations to insects, causing them to "stumble" in flight [2]. We first glue tiny ferromagnetic pins to insects' backs and image their flight using the set-up described above. In Fig. 1d, we show that as a fly crosses the filming volume, the trigger used to initiate recording also activates a pair of magnetic Helmholtz coils that generate a brief magnetic field. Here, the field and pin are both oriented horizontally, so the resulting magnetic torque on the pin reorients the yaw, or heading, of the insect. This experiment can be thought of as an experimental simulation of a disruptive gust of wind. With this technique, however, we have control over the magnitude, direction, and duration of the perturbing torque.

We use Hull Resolution Motion Tracking (HRMT) to extract the wing and body motions from flight videos [1]. We developed this algorithm using computer vision techniques for estimating the shape of a $3 \mathrm{D}$ object from its silhouettes (Fig. 2a-c). For each image, the algorithm first reconstructs the maximal volume $3 \mathrm{D}$ shape that is consistent with the three $2 \mathrm{D}$ shadows captured by the cameras. We find that this shape is sufficiently close to the real insect's shape to allow extraction of coordinates. As shown in Fig. 2d, portions of the hull that correspond to the body, right wing, and left wing are then "dissected" by applying a clustering algorithm that identifies groups of nearby points. Then, we apply a variety of geometric techniques to determine the center of mass position and angular orientation of each group (Fig. 2e, f). For example, using principal components analysis reveals the long axis of each cluster, which identifies the yaw and pitch angles of the body and the stroke and deviation angles for each wing. The wing chord 


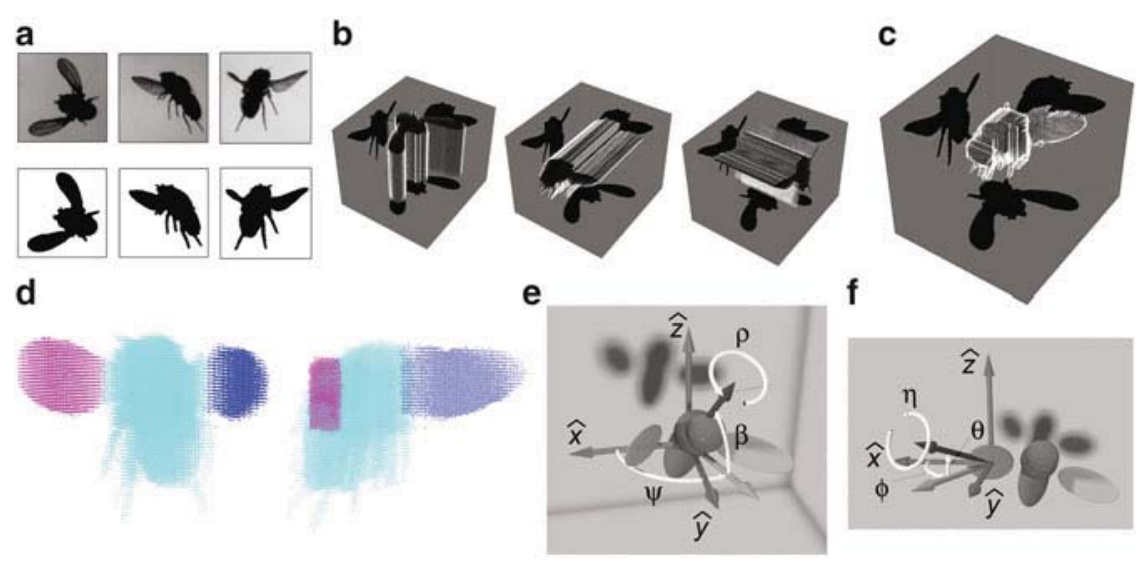

FIG. 2. Hull Reconstruction Motion Tracking (HRMT) [1]. (a) Silhouette information is obtained for each frame of a high-speed video. (b) To construct the visual hull of the insect, each silhouette is extruded into the third dimension. (c) The intersection of these extrusions forms the hull, the maximal volume $3 D$ shape that is consistent with the three $2 D$ shadows. (d) A clustering algorithm 'dissects' the insect into portions corresponding to the body, right wing, and left wing. (e) The body yaw, pitch, roll, and center-of-mass coordinates are extracted by applying geometrical and statistical measures to the body cluster. (f) Similar procedures yield the stroke, deviation, and pitch angles that describe the orientation of each wing

is determined as the diagonal of the wing points, yielding its pitch angle. In the end, the positions and angles of each component are determined for all images, yielding 18 coordinates for each frame of the movie. These data can then be used in further studies, for example, in simulations that predict the aerodynamic forces generated.

3. Simulation Methods: Aerodynamics and Dynamics. Experimental studies of insect flight have been accompanied by theoretical efforts aimed at identifying flapping-wing aerodynamic mechanisms [16-18]. Early studies focused on comparing the forces generated by flapping and flipping insect wings to quasi-steady estimates appropriate for translating wings at fixed orientation [19, 20]. Quasi-steady aerodynamic models approximate the instantaneous fluid forces using a mathematical form that depends on the state variables of the wing, for example, its orientation and velocity. This technique is rapid, tractable, and intuitive, and quasi-steady calculations were able to account for the forces needed to sustain simple flight modes such as level forward motion [19]. However, the general applicability of these early approaches was controversial since the models were not able to account for all experimental observations [20].

These discrepancies inspired researchers to use dynamically-scaled flapping wing models to investigate aerodynamic mechanisms more closely [21-24]. This experimental approach prescribes flapping motions while 

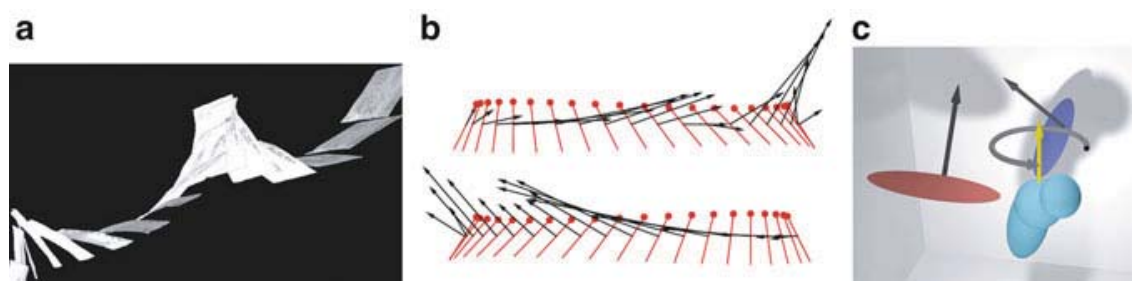

FIG. 3. Flapping-wing aerodynamics at intermediate Reynolds number. (a) Studying fluttering and tumbling plates allows one to extract fluid forces associated with complex motions [32]. A quasi-steady model approximates fluid forces in terms of state variables. (b) Such a model can then be used to estimate the forces on flapping wings [30]. (c) A computational simulation couples a quasi-steady model of aerodynamic forces with the linked rigid-body mechanics of the insect body and wings

measuring fluid forces and visualizing flow structures. These experiments have been used in conjunction with simulations to reveal the influence of unconventional aerodynamic effects, and the most important effect is elevated flight forces that result from the presence of a leading-edge vortex (LEV). The enhanced lift can be achieved either by steadily revolving a wing about a root thereby stabilizing the attached LEV, as is the case for a spinning maple seed [25], or by flipping the wing before the vortex has had enough time to shed [18]. Both mechanisms are expected to be involved in the revolving and flapping motions of insect wings. Mechanized wing experiments have also revealed that other effects associated with wing rotation and wake interference may significantly influence aerodynamic forces $[21,24]$. While these techniques are useful for elucidating basic flappingwing aerodynamics, it has remained challenging to integrate this approach with flight measurements from actual insects [9]. For example, until recently [26], such experiments did not couple the body motion to the forces produced by the wings, an effect that is crucial for understanding the dynamics of free-flight maneuvers [12].

Finally, with the advent of more powerful computers, it has become possible to use computational fluid dynamics (CFD) simulations to numerically solve the Navier-Stokes equations and determine the flight forces and flow structures associated with flapping wings [27-29]. These techniques have been instrumental in showing that flapping flight can be more efficient than fixed-wing flight [30] and in determining the role of wing flexibility [31]. While the CFD approach is promising, it remains computationallyintensive thus prohibiting its use for studies that require the analysis of many wing-strokes. For example, understanding the control of a given flight mode requires many instances of the maneuver each consisting of many wing-strokes [3], and such a statistical analysis remains a challenge for the CFD method.

Our computational approach borrows elements from these different techniques, enabling rapid calculation of flight forces via a quasi-steady 
model that is modified to incorporate unconventional mechanisms (Fig. 3). Specifically, we use a quasi-steady model whose form is distilled from experiments conducted on the fluttering and tumbling of plates [32, 33]. We include the effect of the LEV by using enhanced lift and drag coefficients determined by mechanized wing experiments [24], and the effect of wing flipping is modeled by including a rotational lift term that couples rotational and translational velocities $[32,34]$. Forces are computed for 2D blades and integration over the span of the wing yields an estimate of the 3D force. When compared with CFD calculations and experiments, these quasi-steady estimates have been shown to account for the average forces with an accuracy of about $90 \%[33,34]$.

In order to understand how the forces generated by the wing motions lead to changes in the body position and orientation, we combine the quasisteady aerodynamic calculations with a rigid-body dynamic solver [3, 35]. By solving the Newton-Euler dynamical equations for the coupled wingsbody system, we are able to prescribe wing motions and determine the resulting body motion. Alternatively, the torque at the wing base can be prescribed and the resulting wing and body motions are then computed. These techniques have become increasingly important for elucidating how body motions alter the wing motions relative to the air and hence alter the aerodynamic forces associated with different maneuvers [36-41]. In addition, it has recently become possible to study aspects of free-flight using CFD flow solvers coupled to rigid-body dynamics solvers [42, 43]. We use our quasi-steady implementation in conjunction with the experimental data to analyze the flight dynamics, wing actuation, and sensory-neural control of flying insects.

4. Maneuvering Dynamics. To determine the aerodynamic basis of turns, we develop a systematic procedure for distilling physical mechanisms from kinematic data [3]. We first use our LED arena to elicit turning events, and then reconstruct the maneuver using our HRMT algorithm to extract wing and body kinematics. The results of this procedure for an extreme turn through $120^{\circ}$ performed in $80 \mathrm{~ms}$ are shown in Fig. 4 . To determine the wing kinematics that lead to turning, we use a phaseaveraging algorithm [3] to collapse the data for wing-strokes during the turn. We then examine the measured kinematics in search of differences in the right and left wing motions. Perhaps not surprisingly, during the turn asymmetries appear in all three Euler angles, as shown in Fig. 4ce. To assess which of these changes is aerodynamically important, we form various symmetrized versions of the wing kinematics and play these modified motions in simulation. For example, to assess the importance of the observed changes in stroke angle, we form kinematics that consist of the measured stroke but with the deviation and pitch angles symmetrized to be the mean of those measured for the right and left wings. In this case, the simulated insect fails to turn, indicating that the differences in stroke 

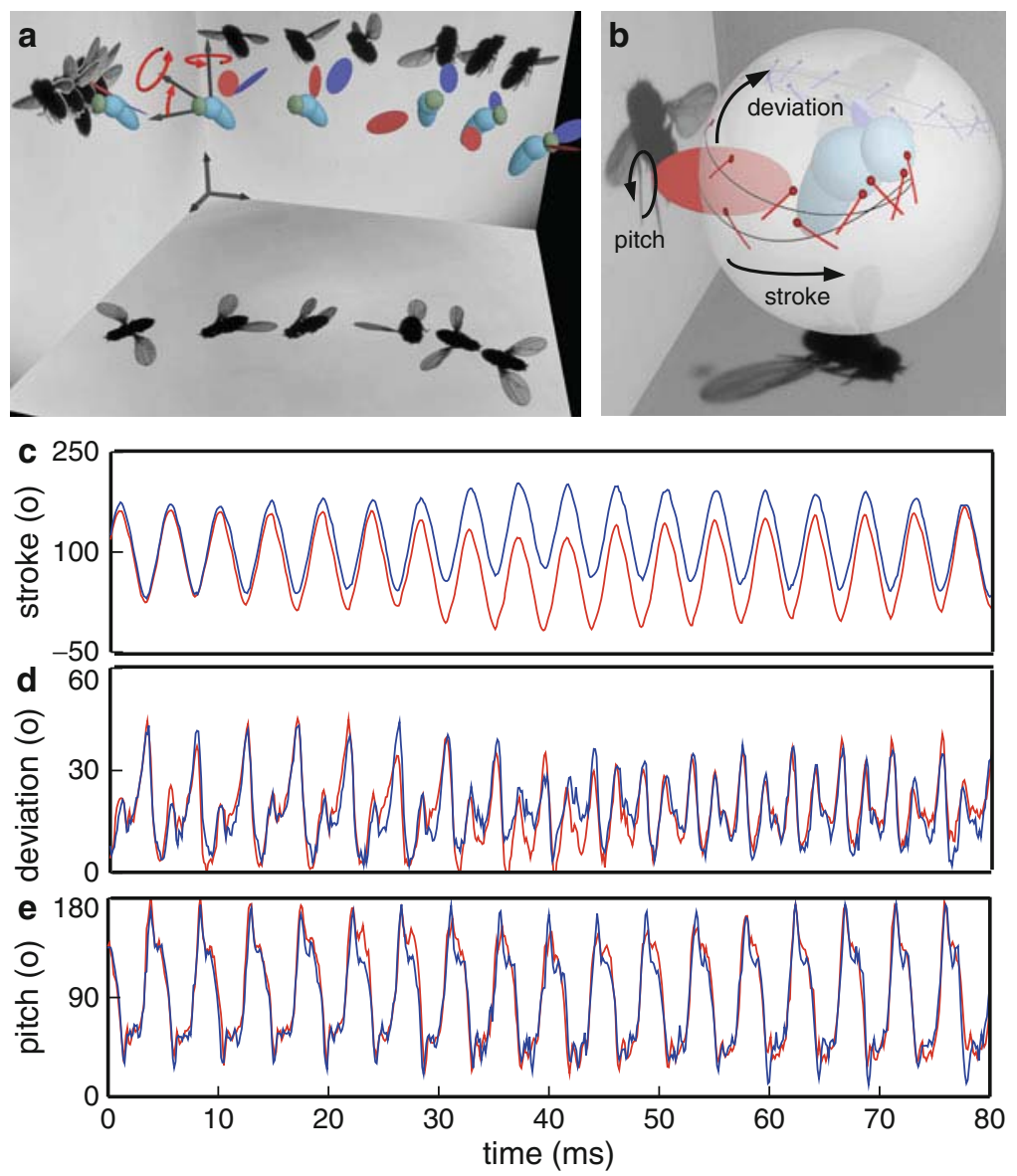

FIG. 4. Body and wing motions during a turning maneuver [3]. (a) The wing and body configuration are captured by the three high-speed cameras, and selected snapshots are displayed on the side panels. We use a motion tracking algorithm to extract the wing and body posture for each frame, and these data are used to render the model insect. The insect is initially hovering on the right, and performs a rightward turn through about $120^{\circ}$ while drifting to the left in this image. (b) Representation of wing motion. Each wing sweeps a path along a globe centered about its root on the body. (c-e) Wing orientation angles throughout the maneuver. Stroke angle is measured in the horizontal plane, deviation angle is the vertical excursion, and pitch angle is measured between the wing chord and the horizontal plane

angle are not crucial to generating yaw torque. Surprisingly, this procedure reveals that small changes in wing pitch are responsible for about $90 \%$ of the yaw torque generated.

For turning maneuvers, the changes in wing pitch correspond to rowing or paddling motions of the wings (Fig. 5a). To turn to the right, the right wing pushes off the air at a high angle of attack on the forward sweep and 

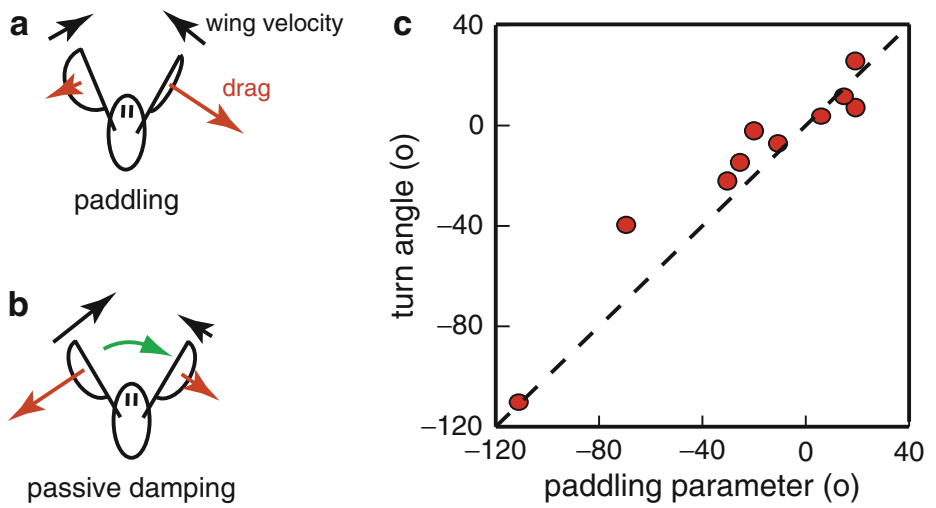

FIG. 5. Minimal model of turning dynamics [2, 3]. (a) Paddling drives turns. In this top-view schematic, a fly turns rightward by inclining its wings at different angles, thus generating differential drag (red arrows) on its wings. (b) Passive damping resists body rotations. As the wings beat during body rotation, their airspeeds are modified and thus induce a net resistive drag. (c) Testing the model. The model predicts that the body turn angle is equal to the paddling parameter, a quantity related to the wing motions. The model is consistent with the measurements of wing and body motions for ten maneuvers. See text for model details

slices through the air at a low attack angle for the backward sweep. This paddling motion generates torque through differential drag. In addition to this driving mechanism, we find that the insect must overcome a resistive aerodynamic torque during its rotation. Here, we build on recent findings that rotations are passively damped during flapping flight $[12,44]$, and we idealize the damping mechanism in Fig. 5b. As the insect rotates, its wings encounter different airspeeds and thus set up resistive aerodynamic forces that oppose the rotation.

These observations can be quantified using a minimal model that incorporates the relevant aerodynamic torques into the Euler equation for yaw rotations,

$$
I \ddot{\psi}=N_{\text {aero }}
$$

where $I$ is the yaw moment of inertia of the insect body and $N_{\text {aero }}$ is the net aerodynamic torque on the insect. The drag on each wing depends on its attack angle $\alpha$ through the drag coefficient, $C_{\mathrm{D}}(\alpha)$, times the square of its speed relative to air. To analyze turning flight by paddling, we consider the case in which the right and left wing angles of attack are different, and each wing beats with average angular speed $\omega$ relative to the body. For an insect body rotating at angular velocity $\dot{\psi}$, the stroke-averaged net aerodynamic torque is found by summing each wing's contribution:

$$
\begin{aligned}
N_{\text {aero }} & \sim-C_{\mathrm{D}}\left(\alpha_{\mathrm{L}}\right) \cdot(\omega+\dot{\psi})^{2}+C_{\mathrm{D}}\left(\alpha_{\mathrm{R}}\right) \cdot(\omega-\dot{\psi})^{2} \\
& \approx-C_{\mathrm{D}}\left(\alpha_{0}\right) \cdot 4 \omega \cdot \dot{\psi}+2 C_{\mathrm{D}}^{\prime}\left(\alpha_{0}\right) \cdot \omega^{2} \cdot \Delta \alpha .
\end{aligned}
$$


Here, we keep leading-order terms in $\dot{\psi}$ and take advantage of the linearity of the coefficient dependence on attack angle [45]: $C_{\mathrm{D}}(\alpha) \approx C_{\mathrm{D}}\left(\alpha_{0}\right)+$ $C_{\mathrm{D}}^{\prime}\left(\alpha_{0}\right) \cdot \Delta \alpha$, where $\alpha_{0}=45^{\circ}, C_{\mathrm{D}}^{\prime}\left(\alpha_{0}\right)$ is the slope, and $\Delta \alpha$ is the mean deviation from $\alpha_{0}$ in attack angle. The aerodynamic torque of Eq. 2 has two components. The first is a damping torque proportional to the yaw velocity $\dot{\psi}$. The second is a torque due to the paddling wing motions that is proportional to the angle of attack difference $\Delta \alpha$. Combining Eqs. 1 and 2, we arrive at the yaw dynamical equation

$$
I \ddot{\psi}=-\beta \dot{\psi}+\gamma \cdot \Delta \alpha .
$$

where the coefficients $\beta$ and $\gamma$ depend on aerodynamic properties of the wings. Thus, the paddling torque combines with damping and inertia to generate the body rotational dynamics.

To provide a test of the aerodynamic model, we derive a prediction for the body turn angle based on the wing motions. Integrating Eq. 3 over the entire turn yields

$$
I \cdot \Delta \dot{\psi}=-\beta \Delta \psi+\gamma \int \mathrm{d} t(\Delta \alpha)
$$

where $\Delta \psi$ and $\Delta \dot{\psi}$ indicate the net change in each quantity. Once the maneuver is complete, the change in yaw velocity $\Delta \dot{\psi}=0$ so that the left hand side of Eq. 4 is eliminated. Solving the remaining portion for the body turn angle yields

$$
\Delta \psi=\frac{\gamma}{\beta} \int \mathrm{d} t(\Delta \alpha)=\omega \int \mathrm{d} t(\Delta \alpha),
$$

where $\omega$ is mean angular speed of the wings relative to the body. Thus, the model predicts that the stronger the paddling and the longer such motions are applied, the greater the body rotates.

To test this prediction, we use our videography apparatus to capture many instances of saccadic turns. We then use our motion tracking algorithm to extract the complete wing and body kinematics for ten such sequences, and use these data to distill the paddling parameter, $\omega \int \mathrm{d} t(\Delta \alpha)$, and the body turn angle, $\Delta \psi$. In Fig. $5 \mathrm{c}$, we plot the body turn angle against the paddling parameter and find that the model captures the overall trend in the relationship. This indicates that paddling and damping are the key physical factors in the dynamics of turning maneuvers. Recently, work on a dynamically-scaled robot has confirmed many of the aerodynamic findings presented here [26].

5. Wing Actuation and Dynamics. At a level deeper, we next investigate how the paddling wing motions themselves arise. Our procedure for investigating wing actuation uses experimental measurements and aerodynamic simulation to extract the torque exerted by the insect on 

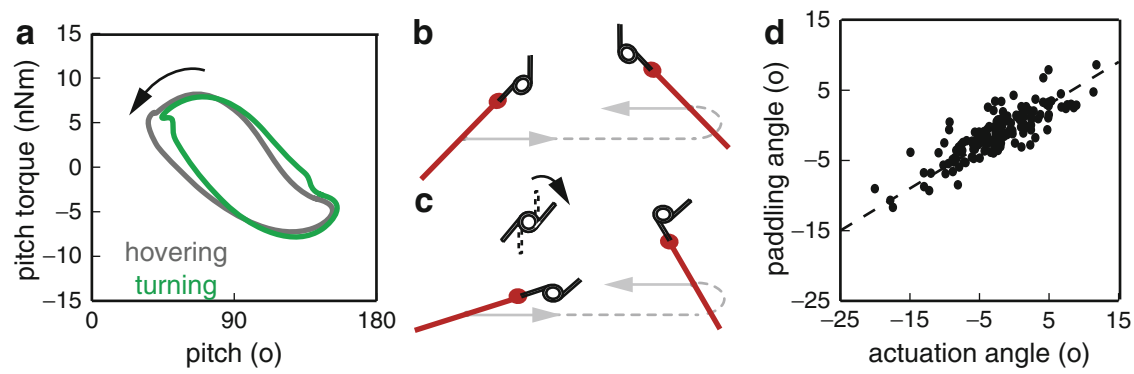

FIG. 6. A biomechanical model of wing actuation [3]. (a) Stress-strain relation for wing pitch. The pitch torque that the insect exerts is plotted against the pitch angle itself. During both hovering and turning flight, the torque exerted by the insect primarily acts like a viscoelastic brake that resists flipping due to inertial and aerodynamic forces. During turning, the mean pitch angle is biased to a pitch angle greater than $90^{\circ}$. $(\boldsymbol{b}-\boldsymbol{c})$ Biomechanical interpretation. The wing hinge or musculature acts like a damped torsional spring that is biased to generate paddling motions. (d) The degree of paddling varies with the bias applied. The paddling angle is approximately one-half the bias angle (dashed line)

its wing [3]. The pitch rotational acceleration is dictated by aerodynamic torque on the wing, the torque exerted by the insect, and the torque associated with driving the wing about an axis above its center of mass. Applying the quasi-steady aerodynamic model to the measured kinematics, we solve for the torque exerted by the insect to pitch the wing. As an analogy to a stress-strain curve or work-loop for a material, this torque can be plotted against the pitch angle itself, as in Fig. 6a. For the symmetric wing motions in hovering and the asymmetric paddling motions, this relation forms a loop which is traversed in a counter-clockwise sense. This direction indicates that work is being done by the fluid to pitch the wing. Even though the insect is actively applying torque to sweep the wings back-and-forth, the wing rotations arise passively.

The difference between wing pitching during hovering and during a turn corresponds to a shift in the loop toward greater values of pitch (Fig. 6a). As discussed above, greater pitch corresponds to paddling motions. To physically interpret the actuation scheme, we note that the derived stress-strain relation is similar to that of a damped torsional spring. The general negative correlation indicates elastic or spring-like behavior, and the open loop indicates damping or viscous-like dissipation. The torque exerted is then well-approximated by $\tau=-\kappa \cdot\left(\eta-\eta_{0}\right)-C \cdot \dot{\eta}$, where $\eta$ is the wing pitch angle, $\kappa$ is the torsional spring constant, $\eta_{0}$ is the rest angle of the spring, and $C$ defines the degree of damping. For hovering, $\Delta \eta_{0}=\eta_{0}-90^{\circ} \approx 0$ so that the wings pitch symmetrically back and forth, as in Fig. 6b. For turning, the spring rest angle is biased such that $\Delta \eta_{0} \neq 0$ and asymmetric paddling motions result, as shown in Fig. 6c. These results suggest a biomechanical interpretation in which the wing musculature and 
hinge act both as a brake that resists wing flipping and as a control lever that dictates the degree of paddling.

This model predicts a linear relationship between the bias of the spring rest angle $\Delta \eta_{0}$ and the paddling angle $\Delta \alpha$ that results: $\Delta \alpha=\mu \cdot \Delta \eta_{0}$ with $\mu=0.6$. We use experimental data to determine the paddling angle and use fits to the torque versus pitch data to determine the bias or actuation angle. In Fig. 6d, we plot the predicted relationship between these quantities as a dashed line, and data points corresponds to both hovering and turning wing-strokes. The theory is able to quantitatively account for the relationship between actuation and resulting wing motion.

Physically, this actuation model can be interpreted as a transmission system that converts flapping motions to flapping-plus-flipping motions. It is an active-passive hybrid system. It is passive in the sense that the insect need not invest power directly to the pitching degree-of-freedom but instead harvests power from the flapping motions. It is active in the sense that pitching motions can be modulated in a simple way to drive maneuvers. The transmission system has other nice properties. First, it eliminates the need to actuate on the time-scale of a single wing-beat, a convenience and perhaps necessity for such animals in which the wing-beat period approaches the fastest neural time-scales. Though the wing motions themselves change within a wing-beat, the actuation need only be applied on the scale of an entire maneuver, say 10-20 wing-beats. Second, the system takes a relatively large actuation to a finer change in wing motion. The data of Fig. $6 \mathrm{~d}$ show that if the base of the wing is biased by $10^{\circ}$ then paddling motions of about $6^{\circ}$ result. This down-gearing may be important considering the precision required for such a sensitive dependence of torque on wing motion modulation.

6. Auto-Stabilization and Sensory-Neural Control. More frequently than performing a turn, fruit flies simply fly forward and maintain their current orientation. However, slight perturbations, such as gusts of wind, will knock them off course. How do these insects maintain their flight course? To explore this issue, we use the magnetic perturbation apparatus to apply torques to free-flying insects [2]. A reconstruction of a typical experiment is shown in Fig. 7a. Remarkably, these insects react to perturbations by quickly recovering their flight heading. In Fig. 7b we show overhead views of this recovery and in (c) we plot the yaw dynamics for this sequence. This example is typical of responses to moderate perturbations in that it is accurate, usually to within a few degrees, and it is fast, usually complete in under 15 wing-beats. To drive this recovery, these insects use paddling wing motions, just as during visually-elicited turning maneuvers. In Fig. $7 \mathrm{~d}$, we quantify these motions by plotting the time-course of the difference between right and left wing attack angles. 
a

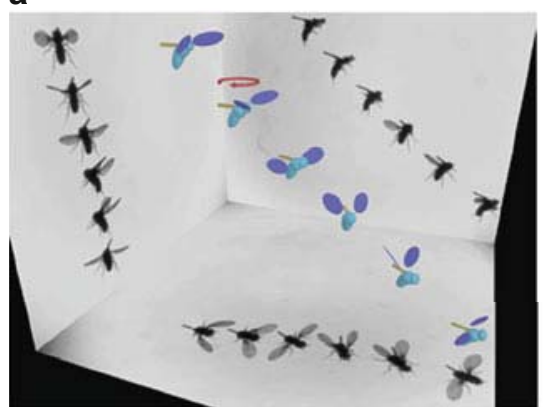

b

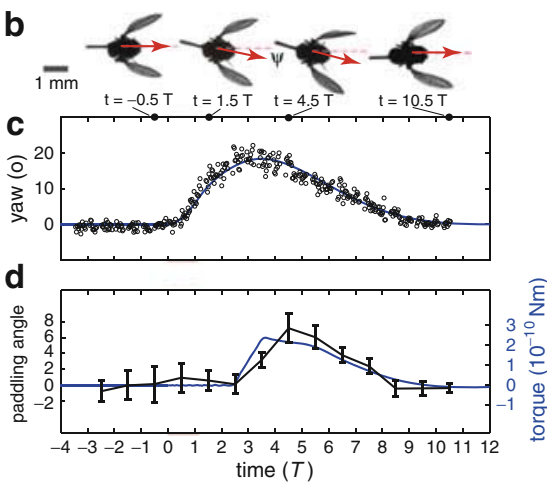

FIG. 7. Flight stabilization in the fruit fly [2]. (a) Reconstruction of a recovery maneuver. The flight of the insect is perturbed by a magnetic torque (red arrow) that is applied for one wing-beat. (b) Top-view stills show the fast and accurate recovery of yaw orientation. (c) Yaw dynamics for an insect perturbed by a magnetic torque (red stripe). (d) Recovery is driven by paddling wing motions which are quantified by the time-course of difference in right and left wing angles of attack. Blue curves in (c) and (d) represent results from a feedback control model

These wing motions must be selected in order to generate accurate recovery of body yaw, and our measurement of both body and wing motions provides a window into this control strategy. A simple strategy would involve the use of sensory measurements of body orientation in order to determine the necessary wing response. A minimal control model [46] that guarantees perfect correction requires that the exerted torque contain a term proportional to the yaw angle $\psi$. However, we find that this socalled proportional $(\mathrm{P})$ controller fails to account for the fast recovery time observed in the flight data. By adding a term that is proportional to the yaw angular velocity $\dot{\psi}$, we arrive at a good match to the yaw data, as shown by the model fit (blue curve) shown in Fig. 7c. This model is a proportional-derivative (PD) scheme [46], and the corrective paddling torque $N_{\text {fly }}=\gamma \cdot \Delta \alpha$ can be written as:

$$
N_{\text {fly }}(t)=K_{\mathrm{P}} \psi(t-\Delta t)+K_{\mathrm{D}} \dot{\psi}(t-\Delta t) .
$$

Here, $K_{\mathrm{P}}$ and $K_{\mathrm{D}}$ are gain constants and $\Delta t$ is the response delay time that we measure to be about three wing-beat periods. This loop delay may reflect both neural latency and inertia of the sensors and motor. In Fig. 7d, we overlay the torque, $N_{\mathrm{fly}}$, predicted by Eq. 6 on the measured $\Delta \alpha$ data and find a strong agreement between the model and experiment.

These findings suggest that these insects sense their body motion and use this information to determine the appropriate response. In fact, flies are equipped with a pair of small vibrating organs called halteres that act as gyroscopic sensors [47]. Anatomical, mechanical, and behavioral evidence indicates that the halteres serve as detectors of body angular 

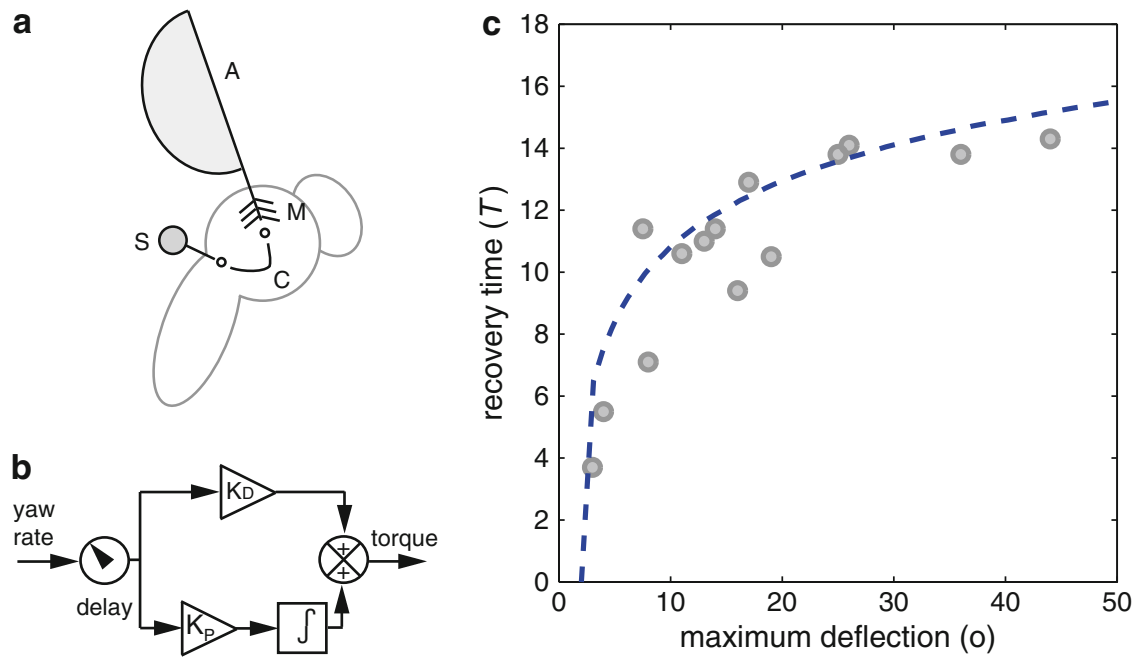

FIG. 8. Feedback control model of yaw stabilization response [2]. (a) Stabilization involves the mechanosensory halteres $(S)$ detecting undesired body rotations, neural circuits $(C)$ processing this information, to drive muscular $(M)$ response and thus aerodynamic (A) torque due to paddling wing motions. (b) The neural controller can be modeled by a yaw and yaw-rate output. (c) The control model accounts for the recovery time taken by the insects in response to perturbations of different strengths

velocity $[47,48]$. These findings suggest that these insects drive their corrective response using an auto-stabilizing feedback loop in which the sensed angular velocity serves as the input to the flight controller. As diagrammed in Fig. 8a, the velocity is sensed by the halteres (S), processed by a neural controller $(\mathrm{C})$, and transmitted by the flight motor $(\mathrm{M})$ into specific wing motions that generate aerodynamic torque (A). In the control diagram of Fig. 8b, the loop is triggered when an external torque induces a yaw velocity that is sensed and processed by neural circuitry to determine the paddling torque $N_{\text {fly }}$.

To test the control model, we use it to predict the total recovery time, $\Delta t_{\text {rec }}$, as a function of perturbation strength. As shown by the dashed blue curve in Fig. 8c, the model predicts that this time rises sharply and then plateaus for increasingly strong imposed deflections. The experimentally measured recovery times confirm this trend, indicating that the control model captures general features of stabilization behavior in fruit flies.

7. Synthesis and Implications. Collectively, these investigations outline the structure of maneuvering and stabilization in the flight of insects. We have put together experimental and analytical techniques that quantify the nature of these flight behaviors and reveal some of the solutions that insects have evolved in interacting with their aerial environment. Having dissected the many levels involved, the next step is to put these 
elements back together. Here, we will outline this synthesis for the specific case of yaw stabilization in fruit flies.

A description of yaw stabilization must include the roles of several key elements: the mechanosensory halteres, neural circuits, wing muscles and hinge, motion of the wings, aerodynamic forces, and the resulting body rotational dynamics. The interaction of these elements forms a closed loop of information flow. At each step in the loop, a given process can be viewed as a transfer function that converts one quantity into another. In the context of our simplest reduced-order models, these transfer functions are linear operators, and the entire feedback loop can be written as a system of linear differential equations that can be summarized by

$$
I \ddot{\psi}=-\beta \dot{\psi}+N_{\text {fly }}+N_{\text {ext }}
$$

where $N_{\text {ext }}$ is the externally-imposed torque and

$$
N_{\text {fly }}(t)=\gamma \cdot \Delta \alpha(t)=\gamma \mu \cdot \Delta \eta_{0}(t)=K_{\mathrm{P}} \psi(t-\Delta t)+K_{\mathrm{D}} \dot{\psi}(t-\Delta t)
$$

is the response torque. Here, we have written the fly's response torque in several equivalent ways to emphasize the roles of the different systems that generate it. At the level of the wing motions, the torque is proportional to the paddling angle $\Delta \alpha$. At the actuation level, the torque is also proportional to the bias angle $\Delta \eta_{0}$. At the sensory and neural levels, the response is proportional to the time-delayed yaw plus yaw-rate.

This study of yaw control should provide a template for future studies aimed at dissecting insect flight behaviors. For the problem of fruit fly flight, the next steps entail devising similar reduced-order models for pitch and roll stabilization. Experiments that apply sequential perturbations could be used to understand the coordination of different degrees-offreedom and may reveal non-linear interactions in the corrective response. Measurements aimed at probing other sensory modalities, such as vision, can be used to elucidate additional feedback circuits [49, 50]. Moreover, experiments that present simultaneous and conflicting stimuli could reveal the interactions between these circuits.

Overall, the combination of carefully-designed experiments and numerical simulations that capture the key physics promise to reveal much about the integration of subsystems in structuring complex flight behaviors. More generally, the organization of processes involved in controlling flight should be shared by a broad array of animal behaviors. As such, we envision that the framework we have outlined here will also provide a useful strategy for unraveling these problems.

Acknowledgements. The work reviewed here was performed by the authors along with K. Coumes and G. Ristroph. We thank the IMA and the organizing committee for putting together a stimulating conference. This paper benefitted from useful discussions with Andy Ruina and Noah Cowan as well as with IMA visitors Russ Tedrake, John Roberts, David Lentink, and Mimi Koehl. 


\section{REFERENCES}

[1] Ristroph L, Berman GJ, Bergou AJ, Wang ZJ, Cohen I (2009) Automated hull reconstruction motion tracking (HRMT) applied to sideways maneuvers of free-flying insects. J Exp Biol 212:1324-1335

[2] Ristroph L, Bergou AJ, Ristroph G, Coumes K, Berman GJ, Guckenheimer J, Wang ZJ, Cohen I (2010) Discovering the flight autostabilizer of fruit flies by inducing aerial stumbles. PNAS 107:4820-4824

[3] Bergou AJ, Ristroph L, Guckenheimer J, Wang ZJ, Cohen I (2010) Fruit flies modulate passive wing pitching to generate in-flight turns. Phys Rev Lett 104:148101

[4] Collett TS, Land MF (1975) Visual control of flight behavior in the hoverfly, Syritta pipiens L. J Comp Physiol A 99:1-66

[5] Reichardt W, Poggio T (1976) Visual control of orientation behavior in the fly. Q Rev Biophys 9:311-375

[6] Mayer M, Vogtmann K, Bausenwein B, Wolf R, Heisenberg M (1988) Flight control during 'free yaw turns' in Drosophila melanogaster. J Comp Physiol A 163:389399

[7] Heisenberg M, Wolf R (1993) The sensory-motor link in motion-dependent flight control of flies. In: Visual motion and its role in the stabilization of gaze. Elsevier, Amsterdam, pp 265-283

[8] Heide G, Goetz KG (1996) Optomotor control of course and altitude in Drosophila is correlated with distinct activities of at least three pairs of steering muscles. J Exp Biol 199:1711-1726

[9] Fry SN, Sayaman R, Dickinson MH (2003) The aerodynamics of free-flight maneuvers of Drosophila. Science 300:495-498

[10] Dickinson MH (2005) The initiation and control of rapid flight maneuvers in fruit flies. Integr Comp Biol 45:274-281

[11] Bender JA, Dickinson MH (2006) Visual stimulation of saccades in magnetically tethered Drosophila. J Exp Biol 209:3170-3182

[12] Hedrick TL, Cheng B, Deng X (2009) Wingbeat time and the scaling of passive rotational damping in flapping flight. Science 324:252-255

[13] Tammero LF, Dickinson MH (2002) The influence of visual landscape on the free flight behavior of the fruit fly Drosophila melanogaster. J Exp Biol 205:327-343

[14] Dickinson MH, Tu MS (1997) The function of Dipteran flight muscle. Comp Biochem Physiol A 116:223-238

[15] Reiser MB, Dickinson MH (2008) A modular display system for insect behavioral response. J Neurosci Methods 167:127-139

[16] Sane SP (2003) The aerodynamics of insect flight. J Exp Biol 206:4191-4208

[17] Lehmann F-O (2004) The mechanisms of lift enhancement in insect flight. Naturwissenschaften 91:101-122

[18] Wang ZJ (2005) Dissecting insect flight. Annu Rev Fluid Mech 37:183-210

[19] Jensen M (1956) Biology and physics of locust flight. III. The aerodynamics of locust flight. Philos Trans R Soc Ser B 239:511-552

[20] Ellington CP (1984) The aerodynamics of hovering insect flight. I. The quasi-steady analysis. Philos Trans R Soc Ser B 305:1-15

[21] Bennett L (1970) Insect flight: lift and the rate of change of incidence. Science 167:177-179

[22] Dickinson MH, Lehmann F-O, Goetz KG (1993) The active control of wing rotation by Drosophila. J Exp Biol 182:173-189

[23] Ellington CP, van den Berg C, Willmott AP, Thomas ALR (1996) Leading-edge vortices in insect flight. Nature 384:626-630

[24] Dickinson MH, Lehmann F-O, Sane S (1999) Wing rotation and the aerodynamic basis of insect flight. Science 284:1954-1960

[25] Lentink D, Dickinson MH (2009) Rotational accelerations stabilize leading edge vortices on revolving fly wings. J Exp Biol 212:2705-2719 
[26] Dickson WB, Polidoro P, Tanner MM, Dickinson MH (2010) A linear systems analysis of the yaw dynamics of a dynamically scaled insect model. J Exp Biol 213:3047-3061

[27] Sun M, Tang J (2002) Unsteady aerodynamic force generation by a model fruit fly wing in flapping motion. J Exp Biol 205:55-70

[28] Ramamurti R, Sandberg WC (2002) A three-dimensional computational study of the aerodynamic mechanisms of insect flight. J Exp Biol 205:1507-1518

[29] Wang ZJ, Birch J, Dickinson MH (2004) Unsteady forces in hovering flight: computation vs experiments. J Exp Biol 207:449

[30] Pesavento U, Wang ZJ (2009) Flapping wing flight can save aerodynamic power compared to steady flight. Phys Rev Lett 103:118102

[31] Young J, Walker SM, Bomphrey RJ, Taylor GK, Thomas ALR (2009) Details of wing design and deformation enhance aerodynamic function and flight efficiency. Science 325:1549-1552

[32] Pesavento U, Wang ZJ (2004) Falling paper: Navier-stokes solutions, model of fluid forces, and center of mass elevation. Phys Rev Lett 93:144501

[33] Andersen A, Pesavento U, Wang ZJ (2005) Unsteady aerodynamics of fluttering and tumbling plates. J Fluid Mech 541:65-90

[34] Sane SP, Dickinson MH (2002) The aerodynamic effects of wing rotation and a revised quasi-steady model of flapping flight. J Exp Biol 205:1087-1096

[35] Featherstone R, Orin D (2000) Robot dynamics: equations and algorithms. In: IEEE international conference robotics \& automation, San Francisco, pp 826834

[36] Deng X, Schenato L, Wu WC, Sastry SS (2006) Flapping flight for biomimetic insects: part I - system modeling. IEEE Trans Robot 22:776-788

[37] Deng X, Schenato L, Sastry SS (2006) Flapping flight for biomimetic insects: part II - flight control design. IEEE Trans Robot 22:789-803

[38] Hedrick TL, Daniel TL (2006) Flight control in the hawkmoth Manduca sexta: the inverse problem of hovering. J Exp Biol 209:3114-3130

[39] Dickson WB, Straw AD, Dickinson MH (2008) Integrative model of Drosophila flight. AIAA J 46:2150-2164

[40] Faruque I, Humbert JS (2010) Dipteran insect flight dynamics. Part 1: longitudinal motion about hover. J Theor Biol 264:538-552

[41] Faruque I, Humbert JS (2010) Dipteran insect flight dynamics. Part 2: lateraldirectional motion about hover. J Theor Biol 265:306-313

[42] Sun M, Wu JH (2003) Aerodynamic force generation and power requirements in forwar flight in a fruit fly with modeled wing motion. J Exp Biol 206:3065-3083

[43] Gao N, Aono H, Liu H (2011) Perturbation analysis of 6DoF flight dynamics and passive dynamic stability of hovering fruit fly Drosophila melanogaster. J Theor Biol 270:98-111

[44] Hesselberg T, Lehmann F-O (2007) Turning behavior depends on frictional damping in the fruit fly Drosophila. J Exp Biol 210:4319-4334

[45] Dickinson MH, Lehmann F-O, Sane S (1999) Wing rotation and the aerodynamic basis of insect flight. Science 284:1954-1960

[46] Bechhoefer J (2005) Feedback for physicists: a tutorial essay on control. Rev Mod Phys 77:783-836

[47] Pringle JWS (1948) The gyroscopic mechanism of the halteres of Diptera. Philos Trans R Soc Lond B 233:347-384

[48] Dickinson MH (1999) Haltere-mediated equilibrium reflexes of the fruit fly, Drosophila melanogaster. Philos Trans R Soc Lond B 354:903-916

[49] Heide G (1983) Neural mechanisms of flight control in Diptera. In: BIONA report 2, Fischer, Stuttgart, pp 35-52

[50] Taylor GK, Krapp HG (2007) Sensory systems and flight stability: what do insects measure and why? Adv Insect Physiol 34:231-316 